\title{
La autorregulación en primaria
}

\section{Óscar García-Gaitero*}

* Magíster en Neuropsicología y Educación. Profesor asociado a la Universidad Unir, Madrid, España. Correo electrónico: oscar.garcia@unir.net

Recibido: 07 de mayo del 2014 Aprobado: 27 de junio del 2014

Cómo citar este artículo: García-Gaitero, Óscar "La autorregulación en primaria". Rastros Rostros 16.30 (2014): 115-118. Impreso. doi: http://dx.doi.org/10.16925/ ra.v16i30.826

\section{Resumen}

La mayor parte de las investigaciones sobre el aprendizaje autorregulado que se han llevado a cabo han sido con estudiantes de secundaria y estudiantes de grados universitarios, y ha olvidado a los alumnos de primaria que son los que más dificultades pueden tener a la hora de aplicar estrategias cognitivas y metacognitivas. El presente artículo destaca la importancia de entrenar a los niños en cómo autorregular su aprendizaje en el comienzo de sus estudios. Ya que en estos primeros años cruciales, los estudiantes aprenden y desarrollan actitudes de autoeficacia que son más fáciles de cambiar que cuando los estudiantes ya han desarrollado y consolidado estilos de aprendizaje.

Palabras clave: alumnos eficientes, aprendizaje autorregulado, aprendizaje activo, desarrollo cognitivo, paradigma.

\section{Self-Regulation in Primary School}

\section{Abstract}

Most research studies on self-regulated learning have been conducted with students in secondary and higher education, forgetting that primary school students are the ones who may face the most problems when cognitive and metacognitive strategies are applied. This article highlights the importance of training children how to self-regulate their learning as they start their studies. In these first crucial years, students learn and develop attitudes of self-sufficiency that are easier to change than when they have already developed and consolidated learning styles.

Keywords: efficient students, self-regulated learning, active learning, cognitive, development, paradigm.

\section{A autorregulação no ensino fundamental}

\section{Resumo}

A maior parte das pesquisas sobre a aprendizagem autorregulada que se realizou foi com estudantes dos últimos anos do ensino fundamental e do ensino médio, e estudantes universitários, e esqueceram os alunos das primeiras séries do ensino fundamental que são os que mais dificuldade podem ter na hora de aplicar estratégias cognitivas e metacognitivas. O presente artigo destaca a importância de treinar as crianças como autorregular sua aprendizagem no começo de seus estudos. Já que nestes primeiros anos cruciais, os estudantes aprendem e desenvolvem atitudes de autoeficácia que são mais fáceis de mudar do que quando os estudantes já desenvolveram e consolidaram estilos de aprendizagem.

Palavras-chave: alunos eficientes, aprendizagem autorregulada, aprendizagem ativa, desenvolvimento cognitivo, paradigma. 


\section{La autorregulación en primaria}

En estos últimos años han aparecido distintos términos para referirse al aprendizaje autorregulado. Entre estos se pueden destacar distintos calificativos, tales como aprendizaje autodirigido, aprendizaje autónomo, aprendizaje independiente, autoaprendizaje. Pero ha sido el término aprendizaje autorregulado (self-regulated learning) el que ha alcanzado una mayor expansión.

La sociedad está experimentando crecimiento y cambios profundos. Entre estos, el rápido desarrollo de las nuevas tecnologías, que exige de nuevos enfoques y principios metodológicos para el manejo, procesamiento y tratamiento de la información. Esto requiere nuevos requisitos relativos a las competencias básicas de los alumnos de nuestra sociedad.

Los cambios en el conocimiento han dado lugar a una gran cantidad de investigaciones sobre cómo hacer el aprendizaje más eficiente. El aprendizaje autorregulado es un concepto que se ha desarrollado durante los últimos 30 años con el fin de satisfacer estas demandas (Winne). Los estudiantes autorregulados disponen de los conocimientos necesarios para aprender de forma efectiva tanto en la escuela como más tarde en la vida. Esto implica un cambio de paradigma en la investigación sobre el aprendizaje y la instrucción, lo que lleva a un enfoque en el cual el estudiante es un agente activo en el proceso de aprendizaje.

La educación actual ha sufrido una serie de transformaciones, al cambiar de paradigma educativo mediante el que guiaba sus acciones $y$, dentro de esta visión, los papeles del profesor y del alumno en el aula se han transformado. En cuanto al profesor, según este nuevo paradigma se le concibe como un mediador, un facilitador, que enseña a pensar, y al estudiante como responsable de su propio aprendizaje, activo, participativo de su proceso de aprendizaje y reflexivo.

Todos estos cambios se han originado en el intento de dar respuesta a las profundas transformaciones, que se vienen dando en la sociedad del conocimiento actual, y en la que se encuentran implicados todos los elementos curriculares de los centros educativos.

Por otro lado, aun cuando los enfoques educativos han venido transformándose, los resultados educativos de los estudiantes siguen reportando un bajo rendimiento final y un elevado porcentaje de suspensos y desmotivación, situaciones que es necesario abordar desde perspectivas diferentes.

Una perspectiva desde la cual es posible estudiar los fenómenos anteriores es la de los diferentes trabajos de autorregulación en el aprendizaje, que se han venido desarrollando durante las dos últimas décadas a lo largo de todo el mundo, y cuyos hallazgos indican una estrecha relación entre las características de los estudiantes que son definidos como autorregulados y sus resultados académicos.

Todos los estudiantes tienen el poder y la habilidad de llegar a ser smart learners, si aprenden o usan distintas técnicas o procesos de autorregulación. Es muy importante que el uso de estas estrategias de autorregulación sea de forma temprana, es decir, desde los primeros cursos de la enseñanza elemental.

Debido a la gran cantidad de evidencia empírica, ahora existe un consenso sobre la eficacia de la autorregulación del aprendizaje para el rendimiento académico (Zimmerman), así como la motivación en el aprendizaje (Pintrich). Además, el aprendizaje autorregulado es un factor clave para el aprendizaje permanente. Proveer a los estudiantes con conocimientos y habilidades sobre cómo autorregular su aprendizaje les ayuda a mejorar su motivación, comportamiento y actividades metacognitivas, con el fin de controlar su aprendizaje (Zimmerman).

Es muy importante diseccionar el concepto de aprendizaje autorregulado y analizar todos sus componentes desde todos los niveles. Por lo tanto, ser un alumno autorregulado implica tener habilidades de estudio, estrategias de aprendizaje, técnicas y habilidades de autorregulación, gran automotivación, interés por la formación permanente, competencia de aprender a aprender, habilidades de pensamiento, estrategias cognitivas, hábitos de estudio, establecimiento de metas, autocontrol, autodeterminación, autogestión y capacidad de organización, entre otras habilidades.

Cada ser humano está llamado al logro de las metas que se ha propuesto y a la adecuada integración al entorno en el que vive, al desarrollo de sus capacidades, de acuerdo a un fin propuesto y a un estilo de vida libremente escogido. Se trata de alcanzar la madurez, ya que: apoderado el hombre de sí mismo es como efectivamente tiene el culmen inevitable de su propia realidad. Vivir es poseer, y la forma suprema de poseer es estar apoderado de sí mismo en un acto de libertad. (Zubiri 153)

$\mathrm{El}$ aprendizaje autorregulado es esa inquietud incansable, ese poder o motivación que debe albergar el alumno y llevarle a buscar siempre la mejor estrategia a la hora de estudiar para conseguir la mayor eficacia en su estudio y ser un alumno elegante y eficiente. 
Esta definición del aprendizaje autorregulado está muy relacionada con la frase de Confucio: "Si das a un hombre un pez, le alimentas un día. Por el contrario si enseñas a un hombre a pescar, le alimentas para toda la vida". Este es el fin último del aprendizaje autorregulado (Alexander y Crutcher).

Por lo tanto, el aprendizaje autorregulado es un proceso de optimización de la persona para el desarrollo de sus capacidades. Ese proceso de optimización debe centrarse, como elemento integrador de todos los demás, en cuatro pilares básicos, tal y como destaca el reciente informe de Delors: aprender a ser, a hacer, a conocer y a convivir. Pilares que también se pueden sintetizar como procesos de humanización y civilización. Aprender es esencialmente construir, representar o esquematizar la realidad; conocer es manipular esas representaciones (Tébar).

El objetivo de esta investigación es determinar las estrategias de autorregulación académica que usan los estudiantes de primaria a través de distintas actividades autorreguladoras.

La importancia de la autorregulación se sustenta en conocer qué tipo de estrategias autorreguladoras (cognitivas y motivacionales) utilizan los estudiantes de primaria para controlar sus pensamientos, motivaciones, conducta y ambiente.

Estos resultados permitirán diagnosticar la situación en la que se encuentra el discente, en cuanto a su proceso de aprendizaje, desde el punto de vista de la autorregulación, en lo que se refiere a las estrategias que se cree tiene que llevar a cabo para lograrlo. De esta forma, tener una visión del proceso de autorregulación del aprendizaje de los estudiantes, permitirá tomar medidas curriculares y administrativas que favorezcan su desarrollo.

Existen dos tipos reconocidos de aprendizajes autorregulados: activo y dinámico. La autorregulación dinámica es un aprendizaje subconsciente, mientras que la autorregulación activa es, para Pintrich: "Consciente, intencional, con esfuerzo, y consiste en la regulación de los recursos atencionales, estrategias cognitivas y metacognitivas y recursos presumiblemente motivacionales, volitivos y de comportamiento".

La presente investigación tiene carácter exploratorio y descriptivo (de la motivación y estrategias de aprendizaje que usan los estudiantes de primaria) así como correlacional (pretende establecer la relación que existe entre el uso de las estrategias y la motivación, de los estudiantes y su rendimiento obtenido durante el curso escolar).
Respecto a los resultados es muy importante que sea revisada la manera en que opera la propuesta teórica en el contexto de las escuelas de primaria en Madrid. Por lo tanto, se sugiere explorar el tema desde el punto de vista de los elementos que lo componen, para determinar la pertinencia de la propuesta teórica en esos escenarios.

Dado que los resultados de un estudio empírico están directamente relacionados con los procedimientos e instrumentos utilizados para operacionalizar un constructo psicológico, se sugiere realizar una revisión teórica e intentar un ejercicio de medición de un constructo complejo pero, al mismo tiempo tan actual, como es el de aprendizaje autorregulado que puede, en primera instancia, parecer inútil, aburrido y extenuante, dado la enorme cantidad de información de que se dispone en la actualidad. Pero es una herramienta para adaptarse a este mundo complejo que es el aprendizaje humano, ya que en la actualidad se viene demandando a los estudiantes en particular y a los seres humanos en general, el uso de estrategias cognitivas que les permitan continuar aprendiendo toda la vida y también estrategias de autorregulación de su propia conducta, que los haga ser sujetos más eficientes en este mundo tan cambiante y lleno de transformaciones.

Por otro lado, ya que en la práctica educativa cotidiana, los profesores pueden fácilmente reconocer a los estudiantes autorregulados, esta situación debe ser aprovechada y capitalizada por los profesores para atreverse a implementar estrategias docentes que permitan a los estudiantes con un desarrollo menor de sus estrategias de autorregulación beneficiarse. Por otro lado, el éxito en el uso de estrategias autorreguladoras requiere retroalimentación de diferentes fuentes, y una de estas, que es fundamental, es el profesor, que cuando conoce y desarrolla los principios del aprendizaje autorregulado abre nuevas posibilidades a sus estudiantes.

El aprendizaje siempre ha sido un tema muy importante de la psicología, lo cual se ha debido a la influencia del conductismo. Tiempo más tarde, con el desarrollo del cognitivismo, se olvidó el tema del aprendizaje. Así, con el paso del tiempo a la consideración de los procesos de pensamiento de los alumnos, en oposición a la enseñanza tradicional, se tuvieron en cuenta una serie de elementos en la mente del alumno que antes no se tenían, tales como, sus conocimientos previos, metas, autoconceptos, estrategias, etc. De esta forma, el proceso de enseñanza/aprendizaje deja de ser un proceso unidireccional, pasivo y de metodología tradicional para convertirse en un proceso activo y significativo en el que el alumno es el agente activo y el verdadero protagonista de su proceso de aprendizaje. 


\section{Referencias}

Alexander, Garrett, y Michael Crutcher. "Functional architecture of basal ganglia circuits: neural substrates of parallel processing." Trends in neurosciences 13.7 (1990): 266-271. Impreso.

Boeakerts, Monique, y Lyn Corno. (2005). "Self-Regulation in the Classroom: A Perspective on Assessment and Intervention". Applied Psychology: and International Review 54.2 (2005): 199-231. Impreso.

Delors, Jacques. La educación encierra un tesoro. Madrid: Santillana-Unesco, 1996. Impreso.

Pintrich, Paul. R. (2000). "Multiple Goals, Multiple Pathways: The Role of Goal Orientation in Learning and Achievement". Journal of Educational Psychology 92.3 (2000): 544-555.
. "A Conceptual Framework for Assessing Motivation and Self-Regulated Learning in College Students". Educational Psychology Review 16.4 (2004): 385-407. Web.

Tébar, Lorenzo. El perfil del profesor mediador. Madrid: Aula Xxi, Santillana, 2003.

Winne, Philip. "Inherent details in self-regulated learning". Educational Psychologist 30.4 (1995): 173-187. Impreso.

Zimmerman, B. J. "Dimensions of academic self-regulation: a conceptual framework for education”. Self-regulation of learning and performance. Eds. D.H. Schunk \& B.J. Zimmerman. Hillsdale: Erbaum, 1994. Impreso.

"Self-regulation involves more than metacognition: A social cognitive perspective". Educational Psychologist 30.4 (1995): 845-862. Impreso.

Zubiri, Xavier. Sobre el sentimiento y la volición. Madrid: Alianza, 1992. Impreso. 